\title{
Modified technique of coronary plaque deformation facilitating side branch entrance in difficult wiring of coronary total occlusion
}

\author{
Wassam ELDin Hadad EL Shafey* \\ Menoufia University Hospital, Egypt
}

\begin{abstract}
The plaque rigidity is an important factor that can complicate proper deployment of coronary stents, consequently increasing the risk of stent thrombosis and restenosis. While aggressive balloon dilation carries the risk of coronary dissections. Thus, pre-treatment of calcific lesions by different modalities of equipment is mandatory to facilitate adequate angioplasty. Here in this case report I utilized the regular balloons with wires to open very tough lesion undilatable with regular PTCA balloons in a step wise technique.
\end{abstract}

\section{Introduction}

One of the major obstacles to the interventional cardiologist, dense calcifications of coronary atheromatous plaque provide an obstacle to successful revascularization [1]. The intravascular ultrasound (IVUS) can improve detection of calcification compared with fluoroscopy alone [2] the rigid plaque can complicate adequate stent deployment including increased risk of in-stent restenosis or stent thrombosis with asymmetric stent expansion [3-5]. while aggressive balloon dilation in noncompliant anatomy can result in coronary dissection [6]. Thus, pretreatment interventions focus on decreasing the degree of calcifications to facilitate adequate angioplasty.

\section{Case report}

Male patient 45 years old, heavy smoker, non-hypertensive, nondiabetic, dyslipidemic presented with angina pain since 2 months, ECG showed infero-lateral ST segment depression, Echo showed inferolateral wall hypokinesia, coronary angiography showed total occlusion of mid large dominant left circumflex artery with very late distal filling. Several attempts were done to wire the lesion, but they were very difficult. After that PTCA was done with up-escalated different sizes semi compliant balloons but the lesion failed to be dilated. Several attempts were done to wire the side branch involved inside the lesion with another wire but were very difficult to reach the entry point.

\section{Discussion and Conclusion}

Many of totally occluded lesions that are undilatable by regular compliantor semi compliant balloons are formed from fibrocalcific plaques; hence they need plaque modification strategies like scoring balloons and rotablators.

To do plaque modification you need an initial step which is a crossed wire through the lesion. In some totally occluded lesions, they may involve sizable side branches with difficult wiring (bifurcation included) so you need to tackle this problem first as to manage the lesion properly. Regular pre-stenting balloon dilatation is mandatory in most of tight lesions as to facilitate the crossing of stents. In some situations, the pre-stenting balloons could be utilized in many traditional and untraditional ways as to overcome difficult lesions including fibrocalcific and heavily calcified plaques (regular and scoring balloons) (Figures 1 and 2) and thrombus containing lesions [7].

Here in current case I utilized regular balloons with different sizes in new way, where I faced a totally occluded bifurcation lesion undilatable with regular PTCA balloons with difficult wiring of the side branch, only the main vessel could be wired. So, i did many tricks as to deform the ostium of the side branch as to be able to wire it.

\section{Technique Steps}

1. Wiring main branch with proper wire.

2. Balloon dilatation with relatively smaller balloons.

3. Another wire parallel to the first one if failed to enter the side branch.

4. Bigger sized short balloon dilatation in the main segment just before side branch entry point proximal optimization technique (POT) fashioned with dilatation against wire facing the expected side branch ostium, this step could act by deforming the plaque involving plaque angle shifting with cutting property of the placed second wire.

5. After that a sizable inflated balloon on the first wire used to trap the distal tip of the second wire inside the distal segment of the main branch, then trying to buckle the second wire into the deformed side branch ostium.

${ }^{\star}$ Correspondence to: Wassam ELDin Hadad EL Shafey, Menoufia University Hospital, Egypt, Tel: 00201006420955; E-mail: dr_wesamhadad@yahoo.com

Key words: undilatable coronary plaques, PTCA, side branch ostial occlusion

Received: November 23, 2019; Accepted: December 11, 2019; Published: December 18, 2019 
Hadad EL Shafey WE (2019) Modified technique of coronary plaque deformation facilitating side branch entrance in difficult wiring of coronary total occlusion

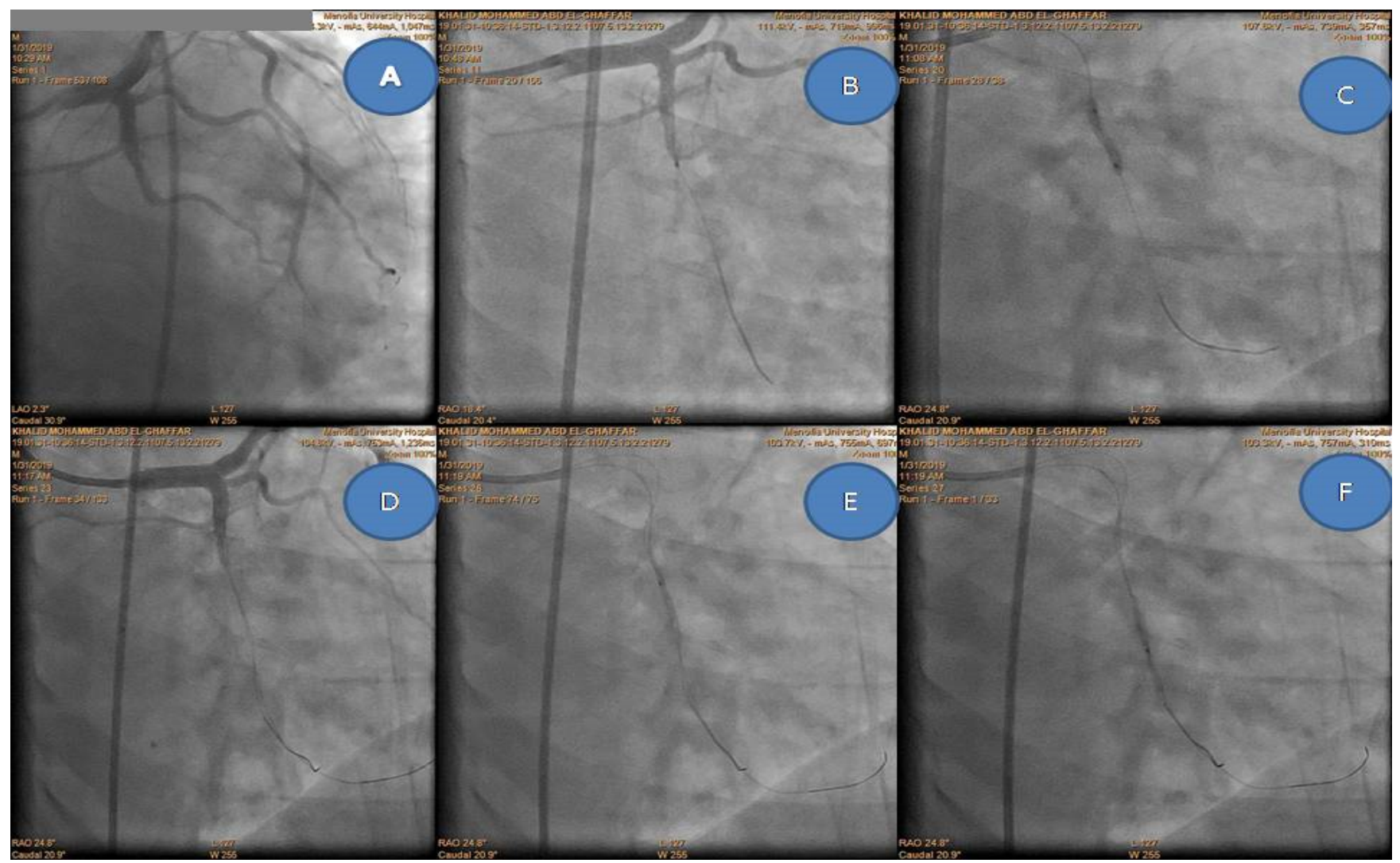

Figure 1. Total occlusion of mid part LCX A, then stepped procedure of deforming the plaque B, C, D, E, F by 2 wires with different balloons sizes and POT fashioned technique

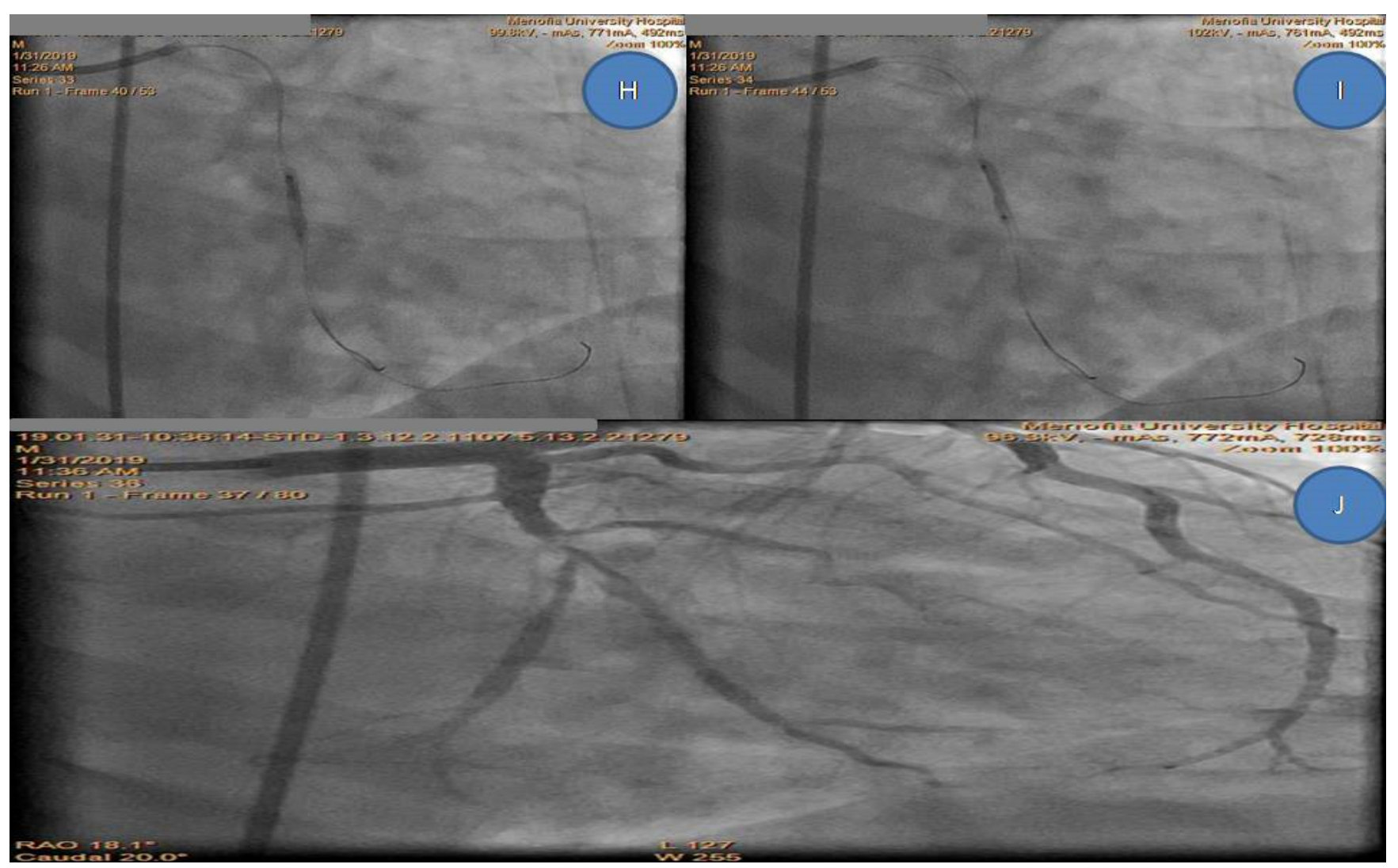

Figure 2. Final steps H, I, J of the technique with complete visualization of the vessel 
6. If succeeded rewire the side branch with the first wire and then pull out the second wire placing it in the distal segment of main branch. (Exchange wires).

Balloon dilatations of the side branch then select the proper bifurcation technique you prefer.

\section{References}

1. Desai R, Mirza O, Martinsen BJ, Kumar G (2018) Plaque modification of severely calcified coronary lesions via orbital atherectomy Single-centre observations from a complex Veterans Affairs cohort. Health Sci Rep 1: e99. [Crossref]

2. Mintz GS, Popma JJ, Pichard AD (1995) Patterns of calcification in coronary artery disease. A statistical analysis of intravascular ultrasound and coronary angiography in 1155 lesions. Circulation 91: 1959-1965. [Crossref]
3. Benezet J, de la Diaz LLS, Cubero JM, Villa M, Fernandez-Quero M, et al. (2011) Drug-eluting stents following rotational atherectomy for heavily calcified coronary lesions: long-term clinical outcomes. J Invasive Cardiol 23: 28-32. [Crossref]

4. Moussa I, Ellis SG, Jones M, Kereiakes DJ, McMartin D, et al. (2005) Impact of coronary culprit lesion calcium in patients undergoing paclitaxel-eluting stent implantation (a TAXUS-IV sub study). Am J Cardiol 96: 1242-1247. [Crossref]

5. Onuma Y, Tanimoto S, Ruygrok P, Neuzner J, Piek JJ, et al. (2010) Efficacy of everolimus eluting stent implantation in patients with calcified coronary culprit lesions: two-year angiographic and three-year clinical results from the SPIRIT II study. Catheter Cardiovasc Interv 76: 634-642. [Crossref]

6. Schlüter M, Cosgrave J, Tübler T, Melzi G, Colombo A, et al. (2007) Rotationa atherectomy to enable sorolimus-eluting stent implantation in calcified, nondilatable de novo coronary artery lesions. Vasc Dis Manag 4: 63-69.

7. Elshafey WEDH (2017) Applicability and reproducibility of ELSHAFEY technique in different thrombus containing coronary lesions. Indian Heart J 69: 528-531. [Crossref]

Copyright: (2019 Hadad EL Shafey WE. This is an open-access article distributed under the terms of the Creative Commons Attribution License, which permits unrestricted use, distribution, and reproduction in any medium, provided the original author and source are credited. 